\title{
Optimization of horizontal well staggered patterns
}

\author{
Zhao Chunsen *, Li Peijing, Guan Dan and Liu Qingjuan \\ Department of Petroleum Engineering, Daqing Petroleum Institute, Daqing, Heilongjiang 163318, China
}

\begin{abstract}
Staggered line-drive patterns are widely used in oilfields. In this paper, to optimize a staggered pattern of horizontal wells, a 3D problem was divided into two 2D ( $x-y$ plane and $y-z$ plane) problems with the pseudo-3D method, conformal transformation and superposition principle. A productivity equation for a horizontal well was deduced, which can be used to optimize the well pattern. A relationship between the length of horizontal wells and the shape factor of well patterns was established. The result shows that optimized well patterns can improve oil production from horizontal wells. This provides a theoretical basis for horizontal well applications to the development of oilfields, especially for overall development of oilfields by horizontal wells.
\end{abstract}

Key words: Horizontal well, productivity calculation, optimization of well pattern

\section{Introduction}

In recent years, horizontal wells have been increasingly used in oil fields worldwide. Meanwhile, great progress has been made in the research into horizontal well technology (Popa and Clipea, 1998; Wan, 1995; Wu et al, 2005; Yao et al, 1997). Optimization of staggered pattern of horizontal wells and vertical wells has been reported (Zhao et al, 2005a). The type of horizontal well patterns directly affects the sweep efficiency of water flooding, but related research and reports are few. This paper discusses the optimization of staggered pattern of horizontal wells, and derives the exact solution to the productivity equation of horizontal wells in staggered patterns with the pseudo-3D method.

\section{Description of well pattern}

A staggered line-drive pattern of horizontal wells is composed of innumerable horizontal well arrays, where the alternate parallel injection arrays and production arrays are staggered. Assume that the effective length of horizontal wells is $2 l$, the oil layer thickness is $h$, and the horizontal wells are drilled in the same horizontal plane and located at the reservoir center in the vertical plane, i.e., at a distance $h / 2$ from the reservoir top to bottom. The distance between adjacent wells in each row is $2 a$ and the row spacing (distance between adjacent rows of injection (or production) wells) is $2 b$, as shown in Fig. 1.

The rows of production wells are located at $\left(x_{0}, y_{0}\right)=(2 a j$, $2 n b), j, n=0, \pm 1, \pm 2, \pm 3, \cdots$; injection well rows are located at $\left(x_{0}, y_{0}\right)=((2 j+1) a,(2 n+1) b), j, n=0, \pm 1, \pm 2, \pm 3, \cdots$. The production and injection rates of horizontal wells are $Q$.

*Corresponding author. email: zhaochunsen@126.com Received January 6, 2008

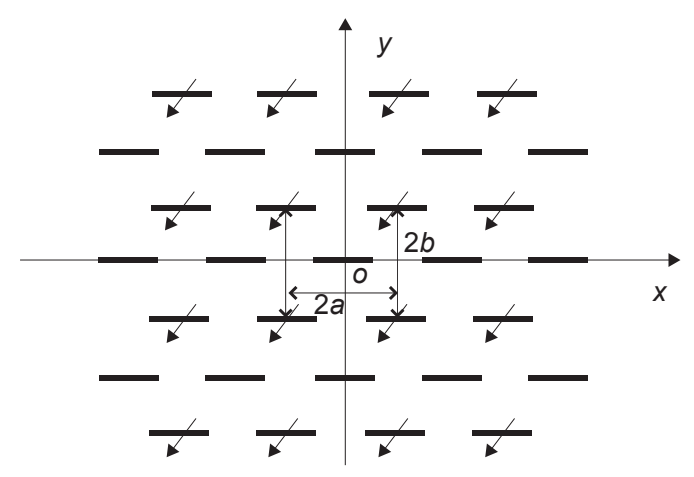

Fig. 1 A staggered line-drive pattern using horizontal wells

\section{Determination of well productivity for a staggered line-drive pattern}

\subsection{Basic idea}

Due to the complexity of fluid flow in reservoirs with horizontal wells in a staggered line-drive pattern, it is very difficult to directly obtain an analytical solution to the productivity equation for a horizontal well in a $3 \mathrm{D}$ space. Therefore, applying the pseudo-3D method, the 3D problem was translated into two $2 \mathrm{D}(x-y$ plane and $y-z$ plane) problems. In the $x-y$ plane, using conformal transformation, the potential distribution of any row of horizontal wells is derived. By adopting the superposition principle, the potential of infinite horizontal well rows at any point in the plane can be obtained, so as to acquire the potential of infinite horizontal well rows at the bottom of injection and production wells. According to the superposition principle, the potential difference between the bottom of the injection well and the bottom of the production well can be obtained. By considering the resistance to flow in the $y-z$ plane in a horizontal well, the exact solution to the productivity equation can be obtained. A computer program was developed to optimize well patterns. 


\subsection{Well productivity}

Assume that the oil layer is homogeneous, with a uniform thickness of $h$, and that single-phase fluid in the oil layer is incompressible. Take any horizontal well as the origin, $x$-axis is the direction of the well row. A row of infinite horizontal wells is shown in Fig. 2. The radius of well is $r_{\mathrm{w}}$.

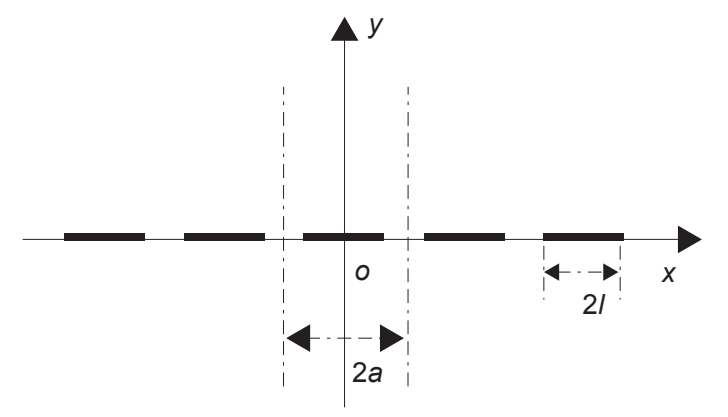

Fig. 2 An infinite row of horizontal wells

By considering the symmetry of horizontal well arrays, the formation can be considered as a separate independent unit, with impermeable boundaries. Therefore, only a single unit need to be analyzed. Hence, the unit included by dashed lines in Fig. 2 was studied. A conformal transformation is made on the unit as follows (Zhao et al, 2005a):

$$
\begin{aligned}
& w_{1}=\sin \frac{\pi}{2 a} z \\
& w=\arcsin \left(w_{1} / \sin \frac{\pi l}{2 a}\right)
\end{aligned}
$$

The potential distribution at any point $(x, y)$ generated by a horizontal well array in the $x$ direction:

$$
\begin{aligned}
& \Phi=\frac{Q}{2 \pi h}|\eta|+c \\
& |\eta|=\frac{1}{\sqrt{2} \sin \frac{\pi l}{2 a}}\left(\sin ^{2} \frac{\pi l}{2 a}+\operatorname{ch}^{2} \frac{\pi}{2 a} y-\cos ^{2} \frac{\pi}{2 a} x+\right.
\end{aligned}
$$

$\left.+\sqrt{\left(\sin ^{2} \frac{\pi l}{2 a}+\operatorname{ch}^{2} \frac{\pi}{2 a} y-\cos ^{2} \frac{\pi}{2 a} x\right)^{2}-4 \sin ^{2} \frac{\pi}{2 a} x \operatorname{ch}^{2} \frac{\pi}{2 a} y \sin ^{2} \frac{\pi l}{2 a}}\right)^{\frac{1}{2}}$

where $c$ is constant; and $Q$ is the production rate of a horizontal well in the $x-y$ plane.

Moreover, the potential at any point $(x, y)$ in infinite horizontal well arrays in the well pattern equals to the superposition of potentials of each well array at this point. If the potential of all horizontal wells at $(x, y)$ in well pattern is $\Phi_{\mathrm{h}}$, then:

$$
\Phi_{\mathrm{h}}=\left.\sum_{n=-\infty}^{n=+\infty} \Phi\right|_{y=(2 n+1) b}=\sum_{n=1}^{n=+\infty}\left(\left.\Phi\right|_{y=(2 n-1) b}+\left.\Phi\right|_{y=-(2 n-1) b}\right)
$$

Potential distributions at production well $(0,0)$ and injection well $(a, b)$, which are generated by infinite horizontal well arrays, can be written as:

$$
\begin{aligned}
\Phi_{\mathrm{hi}}= & \frac{Q}{2 \pi h} \sum_{n=1}^{+\infty} 2 \operatorname{arch} \frac{\operatorname{ch} \frac{2 n-1}{2 a} \pi b}{\sin \frac{\pi l}{2 a}}+c \\
\Phi_{\mathrm{hp}}= & \frac{Q}{2 \pi h} \sum_{n=1}^{\infty}\left[\operatorname{arch} \frac{\left(\sin ^{2} \frac{\pi l}{2 a}+\operatorname{sh}^{2} \frac{n-1}{a} \pi b\right)^{\frac{1}{2}}}{\sin \frac{\pi l}{2 a}}\right. \\
& \left.+\operatorname{arch} \frac{\left(\sin ^{2} \frac{\pi l}{2 a}+\operatorname{sh}^{2} \frac{n}{a} \pi b\right)^{\frac{1}{2}}}{\sin \frac{\pi l}{2 a}}\right]+c
\end{aligned}
$$

Applying the superposition principle and including $\operatorname{arch} \frac{\left(\sin ^{2} x+\operatorname{sh}^{2} y\right)^{\frac{1}{2}}}{\sin x}=\operatorname{arsh} \frac{\operatorname{sh} y}{\sin x}$, the potential difference

can be expressed as follows:

$$
\begin{gathered}
\Delta \Phi=\frac{Q}{2 \pi h}\left[\ln \frac{\operatorname{ch}^{2} \frac{\pi b}{2 a} \operatorname{ch} \frac{3 \pi b}{2 a}}{\operatorname{sh} \frac{\pi r_{\mathrm{w}}}{2 a} \operatorname{sh}^{2} \frac{\pi l}{2 a}}+2 \operatorname{arch} \frac{\operatorname{ch} \frac{\pi b}{2 a}}{\sin \frac{\pi l}{2 a}}\right. \\
\left.2 \operatorname{arch} \frac{\operatorname{ch} \frac{3 \pi b}{2 a}}{\sin \frac{\pi l}{2 a}}-2 \operatorname{arsh} \frac{\operatorname{sh} \frac{\pi b}{a}}{\sin \frac{\pi l}{2 a}}-\operatorname{arsh} \frac{\operatorname{sh} \frac{2 \pi b}{a}}{\sin \frac{\pi l}{2 a}}\right]
\end{gathered}
$$

Let

$$
\begin{aligned}
& \Delta \Phi=\frac{Q}{2 \partial h}\left[\ln \frac{\operatorname{ch}^{2} \frac{\partial b}{2 a} \operatorname{ch} \frac{3 \partial b}{2 a}}{\operatorname{sh} \frac{\partial r_{\mathrm{w}}}{2 a} \operatorname{sh}^{2} \frac{\partial l}{2 a}}+2 \operatorname{arch} \frac{\operatorname{ch} \frac{\partial b}{2 a}}{\sin \frac{\pi l}{2 a}}\right. \\
& \left.2 \operatorname{arch} \frac{\operatorname{ch} \frac{3 \partial b}{2 a}}{\sin \frac{\partial l}{2 a}}-2 \operatorname{arsh} \frac{\operatorname{sh} \frac{\partial b}{a}}{\sin \frac{\partial l}{2 a}}-\operatorname{arsh} \frac{\operatorname{sh} \frac{2 \partial b}{\sin \frac{\partial l}{2 a}}}{\sin }\right]
\end{aligned}
$$

Considering the internal resistance in a horizontal well, we can determine the potential difference of infinite horizontal well arrays between production well $(0,0)$ and injection well $(a, b)$, and the exact solution to the production of horizontal well in a rectangular staggered pattern. 


$$
Q_{\mathrm{h}}=\frac{2 \pi k h \Delta p}{\mu\left(R_{1}+\frac{h}{2 l} \ln \frac{h}{2 \pi r_{\mathrm{w}}}\right)}
$$

where $Q_{\mathrm{h}}$ is the horizontal well production, $k$ is the permeability and $\mu$ is the fluid viscosity.

In order to facilitate analysis, two dimensionless parameters were proposed as follows:

Dimensionless productivity of horizontal well:

$$
Q_{\mathrm{hD}}=\frac{Q_{\mathrm{h}} \mu}{2 \pi k h \Delta p}
$$

Dimensionless length of horizontal well:

$$
l_{\mathrm{D}}=\frac{l^{2}}{a b}
$$

For a normal five-point pattern, $a=b$, $\operatorname{arch} x \approx \ln 2 x$ and $\operatorname{arsh} x \approx \ln 2 x$, so Eq. (7) can be simplified as follows:

$$
\Delta \Phi=\frac{Q}{2 \pi h}\left[\ln \frac{\sqrt{2} a}{r_{\mathrm{w}}}+0.256-\ln \sin \frac{\pi l}{2 a}\right]
$$

By including the internal flow resistance in horizontal wells (Wu and Cao, 2006), the oil production of horizontal well can be rewritten as follows:

$$
Q_{\mathrm{h}}=\frac{2 \pi k h p}{\mu\left(\ln \frac{\sqrt{2} a}{r_{\mathrm{w}}}+0.256-\ln \sin \frac{\pi l}{2 a}+\frac{h}{2 l} \ln \frac{h}{2 \pi r_{\mathrm{w}}}\right)}
$$

\section{Optimization of pattern shape}

The dimensionless productivity of a horizontal well is influenced by its dimensionless length and the shape factor of the well pattern (Joshi, 1988). When the pattern area is fixed and the length of the horizontal well is constant, productivity varies with the change of well pattern shape. Therefore, there must be an optimum pattern shape, with which the maximum productivity could be achieved from the horizontal well pattern with the same pattern area and horizontal well length.

Eq. (8) shows that the oil production from horizontal wells is related to the pattern shape factor $F=a / b$. According to the analysis mentioned above, the optimum shape factor of a rectangular pattern has an exponential relationship with the dimensionless length of the horizontal wells. Therefore, with Eq. (8), we try to seek the expression between the dimensionless length of the horizontal wells and the optimum factor of pattern shape, so as to optimize the well pattern.

\subsection{Relationship between optimum pattern shape factor and dimensionless length of horizontal wells}

With the above methods, a computer program was developed. Considering the reality of an oilfield, the calculation was conducted by taking the pattern area as $22,500,90,000$ and $360,000 \mathrm{~m}^{2}$ and the oil layer thickness as 10,30 and $50 \mathrm{~m}$, respectively. The calculation results of dimensionless length $l_{\mathrm{D}}$ of the horizontal wells and the optimum shape factor $F_{\mathrm{OP}}$ show that:

1) When the dimensionless length $l_{D}$ is specified, there exists a optimum pattern shape factor $F_{\mathrm{OP}}$, with which the dimensionless productivity is maximum.

2) The pattern area and oil layer thickness have a slight influence on $l_{\mathrm{D}}$ and $F_{\mathrm{OP}}$. The relationship between $l_{\mathrm{D}}$ and $F_{\mathrm{OP}}$ does not vary with pattern area and oil layer thickness, as shown in Fig. 3. Therefore, the definition of the dimensionless length of horizontal wells proposed in this paper has significant theoretical value, which provides a basis for the optimization of patterns with different layer thicknesses and pattern areas, and makes the optimization of well pattern simple and easy.

Fig. 3 shows that with increasing dimensionless length of the horizontal wells, the optimum shape factor increases, i.e., the extent of deviation of the well pattern from a square increases. Regression indicates that they satisfy the following expression:

$$
F_{\mathrm{OP}}=0.024 l_{\mathrm{D}}^{2}+0.2612 l_{\mathrm{D}}+1.4478 \quad\left(R^{2}=1\right)
$$

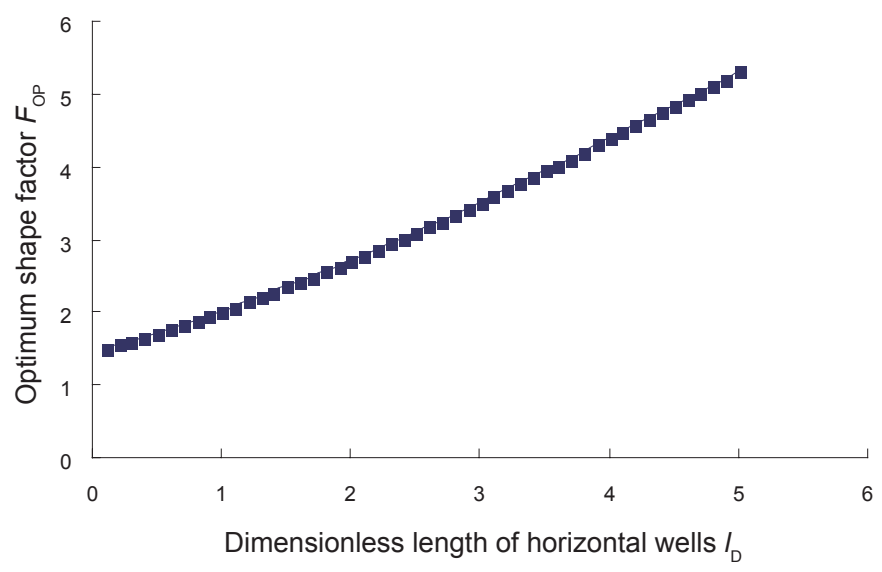

Fig. 3 Relationship between optimum shape factor and dimensionless length of horizontal wells

\subsection{Relationship of optimum dimensionless productivity and dimensionless length of horizontal wells}

For a well pattern with an optimum shape factor, the relationship between the optimum dimensionless productivity and dimensionless length of horizontal wells is shown in Fig. 4. 

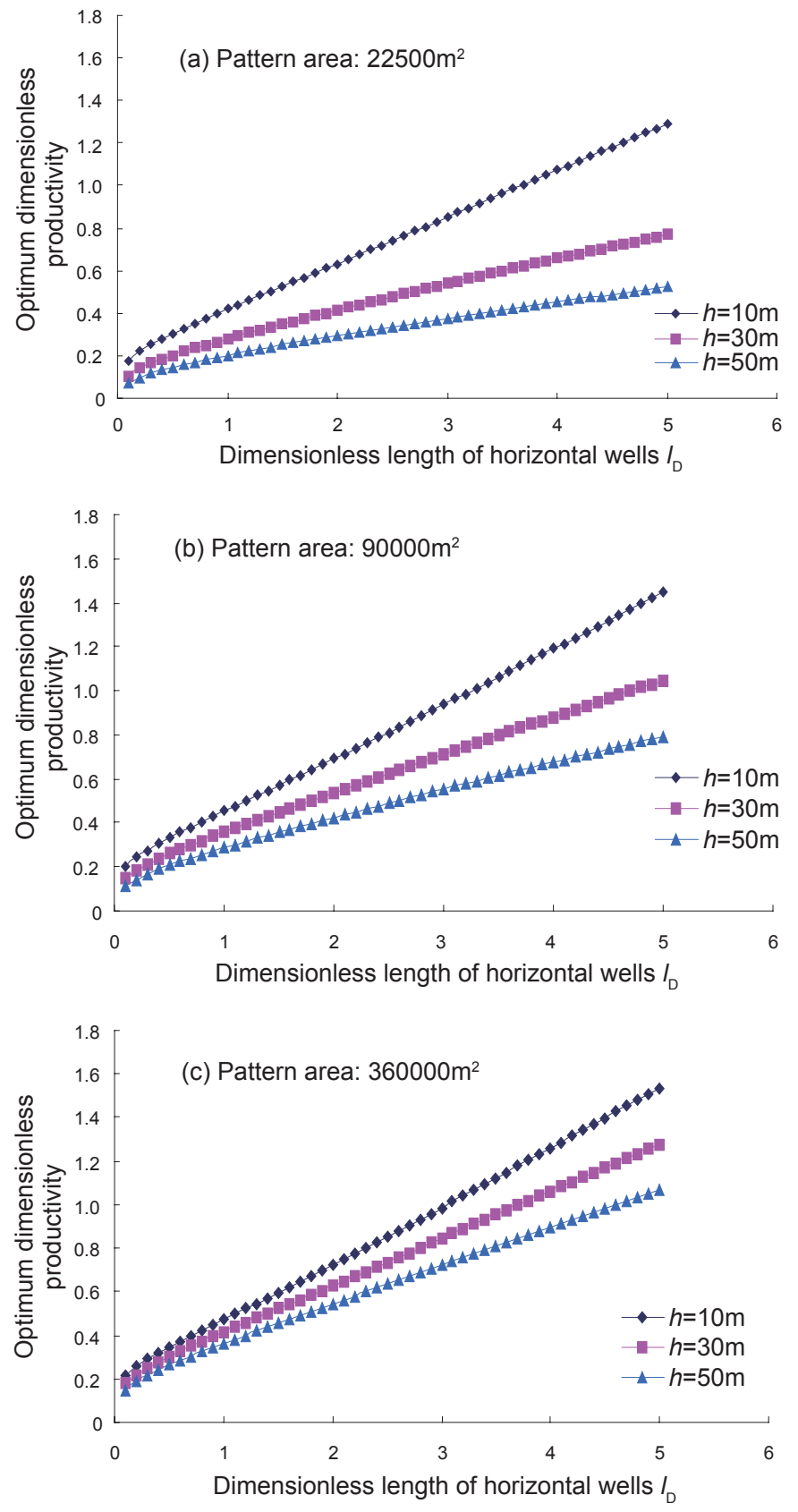

Fig. 4 Relationship between optimum dimensionless productivity and dimensionless length of horizontal wells

For developing oil layers with the same thickness, the optimum dimensionless productivity increases with increasing pattern area. For thin oil layers, the pattern area has little influence on the optimum dimensionless productivity of horizontal wells. The optimum dimensionless productivity of a horizontal well increases quickly with the increase of dimensionless length of horizontal well. For thick oil layers, pattern area has significant effect on the optimum dimensionless productivity of a horizontal well. For a well pattern covering a large area, the effect of dimensionless length on the productivity of horizontal wells becomes obvious. Therefore, adopting a larger well pattern can obtain better performance (Dou, 1999; Zhao et al, 2005b; Zhou et al, 2005).
Therefore, for a flooding pattern of horizontal wells, a well pattern covering a large area and with a long dimensionless length of the horizontal wells is recommended.

\subsection{Influence of an optimized rectangular pattern on production}

Fig. 5 shows the percentage increase in dimensionless productivity of the optimized rectangular pattern compared with that of a square well pattern. Applying the optimized rectangular pattern of horizontal wells can greatly improve well productivity, even to several dozens of percent. For a well pattern covering the same area, the percentage increase in horizontal well productivity becomes larger and larger with the increase of dimensionless horizontal length, especially for thinner oil layers. For the oil layers with the same thickness, the larger the pattern area, the greater the percentage increase of horizontal well dimensionless productivity.
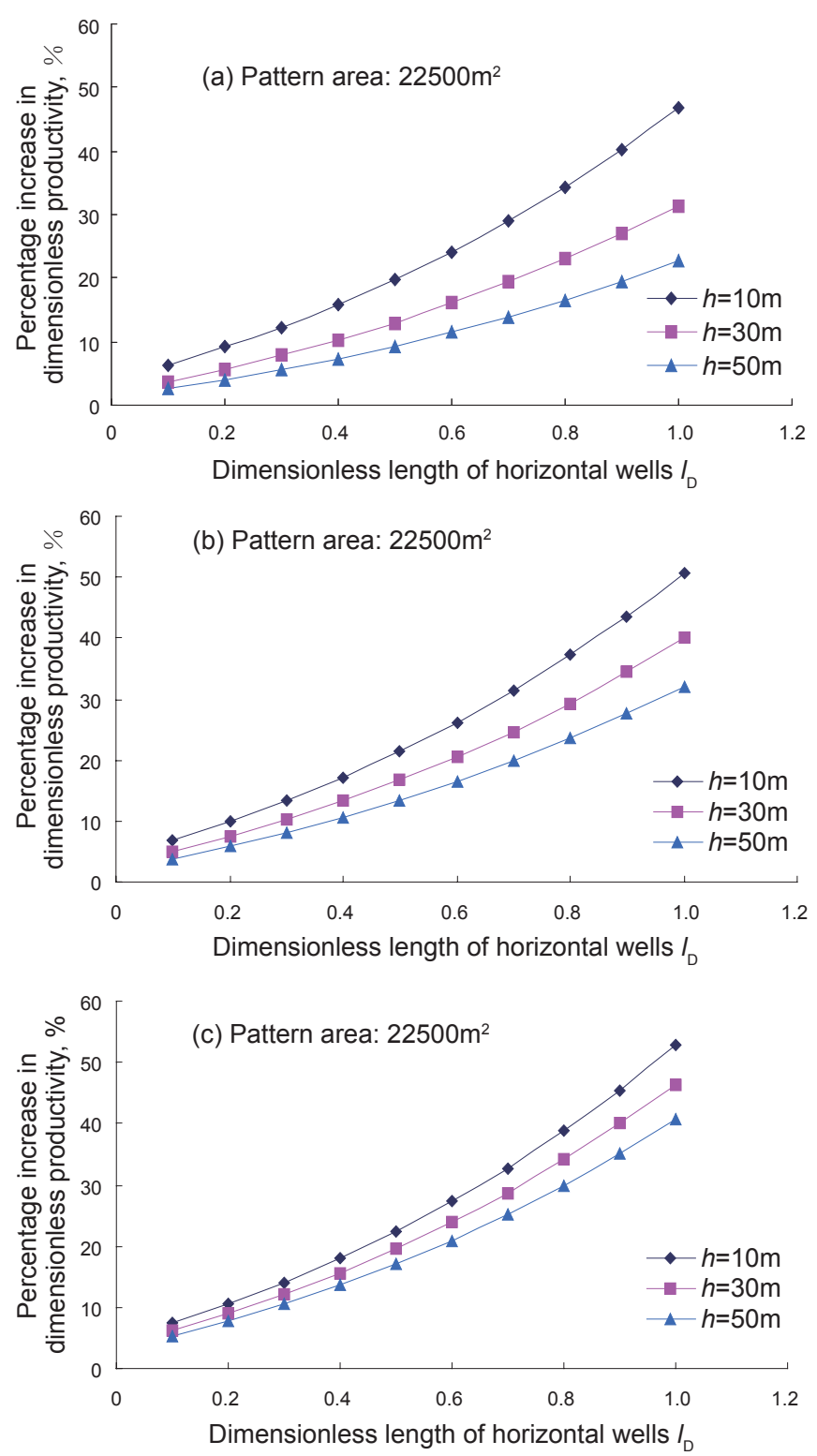

Fig. 5 Percentage increase curve of dimensionless productivity of horizontal wells in the optimized rectangular well pattern 
Therefore for a staggered well pattern, the larger the dimensionless length of horizontal well, i.e. the more severe the extent of well pattern deviates from the square pattern, the greater the increment of well productivity.

\section{Conclusions}

1) The productivity equation of horizontal wells in a staggered rectangular well pattern was established, and the exact analytical solution to this equation was derived with the pseudo-3D method.

2) The relationship between optimum well pattern shape factor and dimensionless length of horizontal well was established. This relationship is independent of pattern area and reservoir (oil layer) thickness. The larger the dimensionless length of horizontal well, i.e. the more severe the deviation of the optimized well pattern from the square pattern, the greater the percentage increase in well productivity. Therefore, a well pattern covering a large area and with a long dimensionless length of horizontal wells is recommended.

\section{References}

Dou H E. Problems concerning the productivity calculation of horizontal wells and branch horizontal wells. Oil Drilling \& Production
Technology. 1999. 21(6): 56-59 (in Chinese)

Joshi S D. Augmentation of well production with slant and horizontal wells. 1988. SPE paper 15375

Popa C G and Clipea M. Improved waterflooding efficiency by horizontal wells. 1998. SPE paper 50400

Wan R P. The technology of horizontal well exploitation. Beijing: Petroleum Industry Press. 1995. 115-160 (in Chinese)

Wu B C, Yao J, Zhang J H, et al. Determination of water breakthrough time in horizontal-vertical well patterns. Acta Petrolei Sinica. 2005. 36(4): 111-114 (in Chinese)

$\mathrm{Wu} \mathrm{J}$ and Cao D Y. Research on 3D distribution of meandering river sand bodies using a sedimentary facies method and 3D geological modeling. China University of Mining and Technology. 2006. 16(3): 349-352

Yao J, Li A F, Chen Y M, et al. Transient pressure analysis for a horizontal well in a box shaped reservoir. Acta Petrolei Sinica. 1997. 18(3): 105-109 (in Chinese)

Zhao C S, Song W L, Sui M Q, et al. Oil-water 2-phase percolation theory for horizontal and vertical well patterns. Acta Petrolei Sinica. 2005a. 26(5): 71-73 (in Chinese)

Zhao C S, Xiao D F, Song W L, et al. Optimization of the staggered well pattern of mixed horizontal and vertical wells. Petroleum Exploration \& Development. 2005b. 32(1): 119-122 (in Chinese)

Zhou D Y, Xiang T W, Feng J L, et al. A simplified approach for determining the reasonable water-free production of horizontal well with bottom water drive reservoir. Acta Petrolei Sinica. 2005. 26(6): 86-89 (in Chinese)

(Edited by Sun Yanhua) 